\title{
Lactate as prognostic factor after out-of-hospital cardiac arrest treated by therapeutic hypothermia
}

\author{
JC Orban ${ }^{1 *}$, M Novain ${ }^{1}$, F Cattet ${ }^{1}$, R Plattier ${ }^{1}$, M Nefzaoui ${ }^{1}$, H Hyvernat ${ }^{2}$, O Raguin ${ }^{3}$, M Kaidomar $^{4}$, C $_{\text {Ichai }}{ }^{1}$ \\ From ESICM LIVES 2015 \\ Berlin, Germany. 3-7 October 2015
}

\section{Introduction}

Out-of-hospital cardiac arrest (OHCA) is a major public health problem whose prognosis is uncertain. Therapeutic hypothermia improved outcome of these patients but also changed the value of several prognosis factors $[1$, 2]. Lactate is a controversial factor associated to outcome after OHCA [3].

\section{Objectives}

Evaluation of lactate as prognosis factor after out-of-hospital cardiac arrest treated by therapeutic hypothermia

\section{Methods}

We performed a retrospective study including adult patients admitted in 4 ICUs for OHCA and treated by therapeutic hypothermia. We collected demographic data, circumstances of cardiac arrest and arterial lactate levels in the first 48 hours after admission. Neurologic outcome was evaluated by the CPC score: favorable outcome (scores 1-2) and unfavorable outcome (scores 35). Data are expressed as median and IQR. Comparisons were made by a Mann-Whitney or a chi-squared tests as appropriate. Odds ratio associated to outcome were estimated by a logistic regression. A p value $<0.05$ was considered as statistically significant.

\section{Results}

Two-hundred and seventy-two patients were included in the study: 89 favorable and 183 unfavorable outcome patients. Favorable outcome patients were younger $(60$ [49-70] vs. 68 [58-76] years; $\mathrm{p}<0.01)$, exhibited shorter durations of no-flow (1 [0-5] vs. 5 [0-10] min; p < 0.01) and low-flow (10 [5-15] vs. 20 [15-30] min; p < 0.01), and a higher proportion of shockable rhythm $(74 \%$ vs. $30 \% ; \mathrm{p}<0.01)$. Lactate levels at different times were lower in the favorable outcome patients (table 1).

In multivariate analysis, several factors were associated to unfavorable outcome: admission lactate levels (OR 1.304 [1.146-1.483] $\mathrm{p}<0.01$ ), age (OR 1.031 [1.01-4.55] $\mathrm{p}<0.01$ ), non-shockable rhythm (OR 1.993 [1.0393.825] $\mathrm{p}<0.01)$. Outcome was different according to quartiles of lactate $(\mathrm{p}<0.01$; figure).

\section{Conclusions}

Lactate levels seem associated to outcome after OHCA treated by therapeutic hypothermia. However, the statistical performance of this test alone is insufficient to establish a neurologic prognosis. Prospective studies associating several parameters are needed to improve prognostication.

Table 1. Lactate levels according to outcome

\begin{tabular}{llllc}
\hline & All patients & Favorable outcome & Unfavorable outcome & p values \\
\hline Lactate H0 & $4.2[2.2-8.1]$ & $2.2[1.5-3.6]$ & $5.4[3.3-9.4]$ & $<0.01$ \\
\hline Lactate H12 & $2.1[1.3-3.4]$ & $1.4[1.0-2.2]$ & $2.5[1.6-4.7]$ & $<0.01$ \\
\hline Lactate H24 & $1.5[1.1-2.4]$ & $1.3[0.9-2.1]$ & $1.8[1.1-2.8]$ & 0.01 \\
\hline Lactate H48 & $1.4[1.1-2.1]$ & $1.3[1.0-1.8]$ & $1.4[1.1-2.5]$ & 0.05 \\
\hline
\end{tabular}

${ }^{1}$ Nice University Hospital, Medical Surgical ICU, Nice, France

Full list of author information is available at the end of the article 


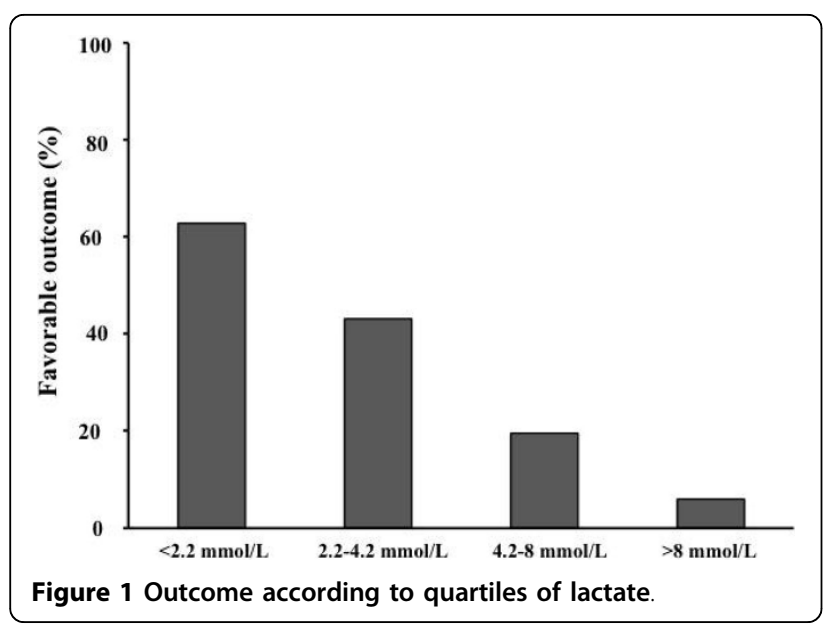

\section{Authors' details}

'Nice University Hospital, Medical Surgical ICU, Nice, France. ${ }^{2}$ Nice University Hospital, Medical ICU, Nice, France. ${ }^{3}$ Antibes General Hospital, Medical Surgical ICU, Antibes, France. ${ }^{4}$ Frejus General Hospital, Medical Surgical ICU, Frejus, France.

Published: 1 October 2015

\section{References}

1. Neurology 2006, 67:203-10.

2. Resuscitation 2010, 81:1219-76.

3. Resuscitation 2013, 84:1078-82.

doi:10.1186/2197-425X-3-S1-A200

Cite this article as: Orban et al:: Lactate as prognostic factor after outof-hospital cardiac arrest treated by therapeutic hypothermia. Intensive Care Medicine Experimental 2015 3(Suppl 1):A200.

\section{Submit your manuscript to a SpringerOpen ${ }^{\odot}$ journal and benefit from:}

- Convenient online submission

- Rigorous peer review

- Immediate publication on acceptance

- Open access: articles freely available online

- High visibility within the field

- Retaining the copyright to your article

Submit your next manuscript at $>$ springeropen.com 